\title{
Velocity-Aware Handover Self-Optimization Management for Next Generation Networks
}

\author{
Abdulraqeb Alhammadi ${ }^{1}$ (), Mardeni Roslee ${ }^{1, *}$, Mohamad Yusoff Alias ${ }^{1}$, Ibraheem Shayea ${ }^{2}$ \\ and Abdullah Alquhali ${ }^{1}$ \\ 1 Faculty of Engineering, Multimedia University, Selangor 63100, Malaysia; \\ abdulraqeb.alhammadi@gmail.com (A.A.); yusoff@mmu.edu.my (M.Y.A.); alhadi1abd@gmail.com (A.A.) \\ 2 Department of Electronics and Communication Engineering, Istanbul Technical University, \\ Istanbul 34467, Turkey; ibr.shayea@gmail.com \\ * Correspondence: mardeni.roslee@mmu.edu.my
}

Received: 9 December 2019; Accepted: 20 January 2020; Published: 17 February 2020

\begin{abstract}
The fifth generation (5G) network is an upcoming standard for wireless communications that coexists with the current $4 \mathrm{G}$ network to increase the throughput. The deployment of ultra-dense small cells (UDSC) over a macro-cell layer yields multi-tier networks, which are known as heterogeneous networks (HetNets). HetNets play a key role in the cellular network to provide services to numerous users. However, the number of handovers (HOs) and radio link failure (RLF) greatly increase due to the increase in the UDSC in the network. Therefore, mobility management becomes a very important function in a self-organizing network to improve the system performance. In this paper, we propose a velocity-based self-optimization algorithm to adjust the $\mathrm{HO}$ control parameters in $4 \mathrm{G} / 5 \mathrm{G}$ networks. The proposed algorithm utilizes the user's received power and speed to adjust the $\mathrm{HO}$ margin and the time to trigger during the user's mobility in the network. Simulation results demonstrate that the proposed algorithm achieves a remarkable reduction in the rate of ping-pong HOs and RLF compared with other existing algorithms, thereby outperforming such algorithms by an average of more than $70 \%$ for all $\mathrm{HO}$ performance metrics.
\end{abstract}

Keywords: handover; HetNets; mobility management; self-optimization

\section{Introduction}

Heterogeneous networks (HetNets) have gained considerable attention in the past few years. These networks consist of different types of cells, such as macro, pico, and femto, which are introduced to meet user demand. As stated by the third generation partnership project (3GPP), the main role of the HetNets is to improve the network performance in terms of boosting capacity and coverage [1]. In next generation cellular networks, an ultra-dense small cell, which comprises numerous small cells overlapped with macro cells, is introduced to boost coverage and improve user experience [2]. Non-standalone (NSA) and standalone (SA) are two road maps of transition from 4G to 5G mobile networks [3]. In the former, the $5 \mathrm{G}$ networks are deployed along with the existing $4 \mathrm{G}$ core network. Thus, the first stage of providing the $5 \mathrm{G}$ service starts with NSA. Then, 5G SA is implemented after the $5 \mathrm{G}$ coverage is completely established. Implementing a $5 \mathrm{G}$ network that overlaps with the $4 \mathrm{G}$ network leads to mobility issues when the users move from one base station (BS) to another $[4,5]$. Mobility management in HetNets is complex due to several types of inter-frequency technologies involved, which requires the user to perform the handover $(\mathrm{HO})$ process while moving from one BS to another. Although the mobility robustness optimization (MRO) function can improve the user mobility experience in $4 \mathrm{G}$ networks, further improvement is necessary to address and resolve the issues of the $\mathrm{HO}$ probability (HOP), HO ping-pong (HOPP), and HO failure (HOF). The improvement 
can be implemented by optimizing the values of the $\mathrm{HO}$ control parameters (HCPs) according to user experience. Mobility management should be properly addressed to avoid service degradation due to high rates of HO, HOPP, and radio link failure (RLF). Furthermore, this issue should be resolved to ensure that the $5 \mathrm{G}$ of cellular networks can provide a seamless communication during user movement among different deployment scenarios [6,7]. Several functions are introduced in self-optimization networks, such as MRO and load balancing optimization [8]. Both functions perform optimization to achieve different objectives during user's mobility and aim to dynamically optimize the HCP values to handle different $\mathrm{HO}$ problems. For example, the MRO function automatically adjusts the HCP values to maintain system quality and performs automatic optimization for HCPs with minimal human intervention. The HCP consists of two main parameters: HO margin (HOM) and time to trigger (TTT). By adjusting these parameters to the proper values during user movements in cell coverage, the rates of HOPP and HOF are minimized, consequently improving the service quality.

Numerous studies have proposed different algorithms to address and solve the HO issue in HetNets. Ni et al. [9] have optimized HCPs based on user equipment (UE) velocity and HO types using mobility state estimation (MSE). In this work, the authors only updated the TTT based on the user velocity with limited updating values of TTT. This method did not fully optimize the HO performance since the gap between these updating values is very big and only three fixed values are selected in accordance with UE velocity. Similarly, Tiwari and Deshmukh [10] have presented an HO decision strategy and an MSE scheme to avoid unnecessary HOs (UHOs) and service failures in HetNets. The proposed model utilizes the number of HOs and sojourn time measurements to estimate the UE velocity. The simulation results show that the proposed MSE model reduces the number of UHOs and service failures. However, the performance with respect to other HO performance metrics, such as HOPP, RLF, and delay, have not been discussed. Researchers [11] have also introduced an adaptive algorithm that selects different values of HOM and load balancing for each UE in HetNets. The $\mathrm{HO}$ decision in the proposed algorithm depends on the signal-to-interference-plus-noise-ratio (SINR) rather than on the received signal strength indicator, which is then used to calculate the actual level of the HOM. Shayea et al. [12-14] have proposed an HO optimization technique based on a weighted function for carrier aggregation. The proposed algorithm automatically adjusts the values of the HOM in accordance with three functions: SINR, traffic load, and velocity. The simulation results have demonstrated that the proposed algorithm enhances the system performance in terms of spectral efficiency at cell edge and outage probability.

Three types of HCPs are considered in this work: TTT, measurement interval, and hysteresis, all of which are adjusted according to the number of HOPP performed in a measurement interval. A three-layer filter technique is applied to improve the HO performance, which requires information about the mobility condition and speed state of UE. Su et al. [15] have adopted a comparison method that compares two parameters, namely, cell boundary crossings and HO execution, to optimize the overall network performance. The HO decision on a target cell is completely dependent on the signal strength measurement. Saeed et al. [16] have developed a model to optimize HOM based on fuzzy logic for HetNets. The fuzzy logic consists of two inputs: call drop rate and load balancing index, both of which adapt the HOM for macro and small cells. In other work [17], the reinforcement learning concept is used to detect an HO in the network. Effective session HOs lead to low drop call rates and also reduce the HOF and HOPP. However, this technique only supports the UE mobility speed up to $120 \mathrm{~km} / \mathrm{h}$. In [18-20], several algorithms have been proposed to investigate and evaluate the issue of mobility management in different mobile speed scenarios. Three types of $\mathrm{HO}$ are considered to adapt the HCPs: too early, too late, and HO to wrong cells. The results demonstrated that the adapted HCPs reduce the rates of HOPP, HOP, and RLF.

The major contributions of this paper can be summarized as follows. First, we formulate and address the issue of MRO in HetNets by focusing on two performance main metrics: RLF and HOPP, which lead to service interruption. Then, we propose a velocity-based optimization algorithm to continuously adjust the HCPs based on the condition of UE. Finally, we evaluate and compare the 
performance of the proposed algorithm to other existing related works. The remainder of this paper is organized as follows. Section 2 explains the system model that used for this work. The proposed velocity-based algorithm is presented in Section 3. Section 4 presents the simulation and performance evaluation. The conclusions are provided in Section 5.

\section{System Model}

We consider a HetNet architecture that comprises several 4G macro cells and a 5G small cell BS where three small cells are uniformly distributed in each macro BS. Each macro BS has a three-sectored hexagonal layout, where each of the sectors operates as an individual cell and the small BSs are omni-directional single-sector cells. Figure 1 displays an example of the HetNets deployment scenario with three small cells placed on one macro cell. $R$ and $r$ represent the radius of macro and small cells, respectively.

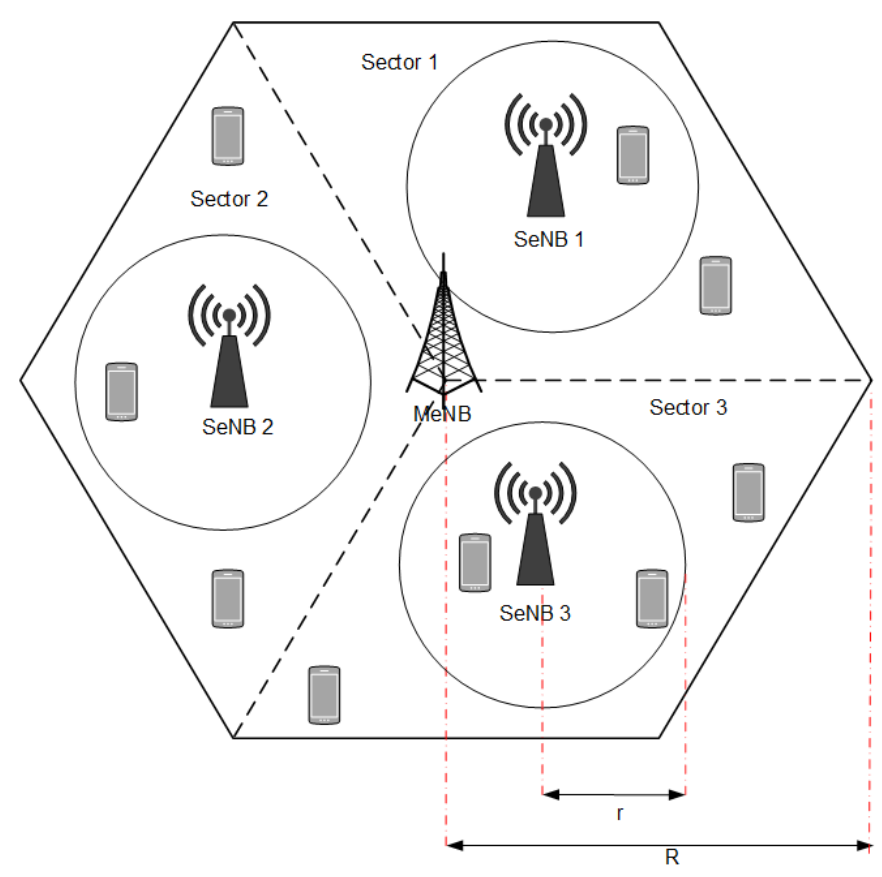

Figure 1. System model for HetNets.

The macro and small BSs operate at low and high frequency bands, receptivity, with reuse frequency factor being equal to one. Table 1 lists the notations used in this paper. The set of macro and small cells are defined as $N_{k}=1, \ldots, N_{m}, N_{l}=1, \ldots, N_{n}$, respectively. The user set is denoted as $N_{u}$ where $U=1, \ldots, U$, where $U$ is randomly distributed in the network with a random mobility model. The UEs receive requested traffic through either the macro or small cells. At each small and macro cell, a distributed self-organizing network collects $\mathrm{HO}$ information and optimizes HCPs. The execution of the HO procedure initializes when a UE moves from a serving cell to a target cell in the same or different network. The serving cell makes an HO decision based on the measurement report (MR) from the UE to begin the HO process to a target cell. The path loss model for different bands in the urban area between a BS, and the user is expressed as [21]:

$$
P L_{u, k, l}=20 \log _{10}\left(\frac{4 \pi r_{0}}{\lambda_{l}}\right)+20 \log _{10}\left(\frac{d_{u, k}}{d_{0}}\right)+\chi,
$$

where:

$$
B S= \begin{cases}\text { small cell } & \text { if } \quad l=1 \\ \text { macro cell } & \text { otherwise }\end{cases}
$$


where $d_{0}$ and $d_{u, k, l}$ represent the reference distance and the distance between the user $U$ and BS $k$, respectively $\left(d_{u, k, l} \geq d_{0} ; d_{0}\right.$ is assumed to be $\left.50 \mathrm{~m}\right) . \lambda_{l}$ is the wavelength at the carrier frequency $f_{c, l} \cdot \chi$ is a Gaussian random variable with zero mean and variance $\sigma^{2}$.

The maximum quality of service (QoS) requirements limit the interference by reducing the RLF. The performance of each UE $u$ should meet the minimum data rate requirement for QoS satisfaction. For channel modeling, the SINR experienced by UE $u$ is modeled as [22,23]:

$$
\operatorname{SINR}_{u, k, l}=\frac{p_{u, k, l} g_{u, k, l} b_{i j}}{\sum_{i \in K \backslash\{k\}} \sum_{j \in U \backslash\{u\}} p_{i j} g_{u, k, l}+P_{A W G N}},
$$

where $p_{u, k, l}$ is the received signal power at $u . g_{u, k, l}$ is the channel gain experienced by UE $u$ at $k . b_{i j}$ is the binary association indicator of user $u\left(b_{i j}=1\right.$ indicates that user $u$ associates with one BS. Otherwise, $b_{i j}=0$.). $p_{i j}$ represents the interference of the received signal power by UE $u$ at $k . P_{A W G N}$ is the additive white Gaussian noise power.

Table 1. List of notations.

\begin{tabular}{ll}
\hline Notation & Description \\
\hline$N_{u}$ & Set of UE $U \in 1,2, \ldots,|U|$ \\
$N_{k}$ & Number of macro BSs \\
$N_{l}$ & Number of small BSs \\
$V_{u}$ & Velocity for UE $u$ \\
$P L_{u, k, l}$ & Path loss model \\
$d_{0}$ & Reference distance between UE $u$ and BS \\
$d_{u}, k, l$ & Distance between UE $u$ and BS $k$ \\
$\lambda_{l}$ & Wavelength at carrier frequency $f_{c, l}$ \\
$\chi$ & Gaussian random variable with zero \\
$S I N R_{u, k, l}$ & mean and variance $\sigma^{2}$ \\
$b_{i j}$ & BinR experienced by UE $u$ at BS $k$ \\
$g_{u, k, l}$ & Channel gain for UE $u$ at BS $k$ \\
$p_{u, k, l}$ & Received signal power by UE $u$ at BS $k$ \\
$P_{A W G N}$ & AWGN power \\
$\theta_{t h}$ & Threshold level for HO decision \\
$V_{u, t}$ & UEu Speed level at time $t$ \\
$V_{r}$ & Reference speed (medium speed)for entire simulation \\
$R S R P_{S}$ & Serving RSRP UE $u$ at BS $k$ \\
$R S R P_{t}$ & Target RSRP for UE $u$ at BS $k$ \\
$\Delta \mathcal{H} \mathcal{O} \mathcal{M}_{u, t}$ & Updated HOM for each UE $u$ at time $t$ \\
$\Delta \mathcal{T} \mathcal{T} \mathcal{T}_{u, t}$ & Updated TTT for each UE $u$ at time $t$ \\
$N_{F}$ & Number of failure HO \\
$N_{C}$ & Number of successful HO \\
\hline &
\end{tabular}

\section{Velocity-Based Optimization Algorithm}

\subsection{HO Problem}

Improper configurations of HCPs increase the rate of RLF, thereby degrading the system performance. The high and low values of HCPs cause too late and too early HOs, respectively. Thus, the HCPs should be frequently adjusted according to the UE's mobility. In this work, the adjustment of the HCPs depends on the UE's speed and the reference signal received power (RSRP), which causes high HOPP and RLF. For example, when a user moves at high speeds, numerous cells (macro or small cells) will be crossed. In this case, the value of the HCPs should be decreased to avoid too late HO. By contrast, when a user moves at low speeds, it experiences a short distance movement and attains a good signal quality; thus, high values of HCPs are required to avoid too early $\mathrm{HO}$. The objective of 
this work is to minimize the probability of occurrence of RLFs and HOPP during the HO processes by adjusting the HCPs, which can be mathematically expressed as:

$$
\begin{array}{r}
\underset{\mathcal{T} \mathcal{T} \mathcal{T}, \mathcal{H O M}}{\operatorname{argmin}} P(\mathcal{T} \mathcal{T} \mathcal{T}, \mathcal{H O M}) \\
\text { Subject } \quad \text { to }: \quad \sum_{j=1}^{N} b_{i j}=1, \forall_{j} \\
T T T_{\min } \leq \mathcal{T} \mathcal{T} \mathcal{T} \leq T T T_{\max } \quad T T T \in(0, \ldots, 5260 \mathrm{~ms}) \\
H O M_{\min } \leq \mathcal{H O} \mathcal{M} \leq H O M_{\max } \quad H O M \in(0, \ldots ., 10 \mathrm{dm}) \\
v_{\min } \leq v_{u, t} \leq v_{\max } \\
b_{i j} \in 0,1, \forall_{i j}
\end{array}
$$

where $P$ refers to the resulting probability of HOPP, RLF, and HOF, which control by a proper selection of $\mathcal{T} \mathcal{T} \mathcal{T}$ and $\mathcal{H O M}$. Constraint (3b) ensures each monitoring user $U$ is associated with one BS $k$; (3c) and (3d) ensure that the adjusted values of $\mathcal{T} \mathcal{T} \mathcal{T}$ and $\mathcal{H O M}$ are within the bounded range. (3e) ensures the UE speed at time $v_{u, t}$ within the bounded range $\left(v_{\min }, v_{\max }\right)$. The last constraint is (3f), which refers to binary constraint that involves exactly two variables to indicate the user association. The procedure HO begins after the serving BS receives the MRs from a UE that makes the HO decision. The UE periodically measures the RSRPs of all serving BSs (every $50 \mathrm{~ms}$ ) and reports a measurement-triggered $\mathrm{HO}$ if a certain condition is satisfied. In this work, all considered events in the 3GPP TR 36.331 are configured for measurement reporting to simulate the network as a realistic environment [24]. The A3 event is triggered when a serving BS becomes worse than the target BS on the basis of a certain margin level. The suitable instance for the UE to report the measurements is immediately after the expiration of the TTT timer. The condition is described as:

$$
R S R P_{S}>R S R P_{T}+\mathcal{H O} \mathcal{M}_{S \rightarrow T}
$$

where $R S R P_{S}$ and $R S R P_{T}$ are the averaged values RSRP measured for serving BS and target BS respectively. $\mathcal{H O} \mathcal{M}_{S \rightarrow T}$ is the $\mathrm{HOM}$ from serving BS to target BS.

\subsection{Proposed Algorithm}

To address the HO optimization, we propose a distributed velocity-based optimization algorithm to enhance the system performance. The proposed algorithm is an enhancement of our previous work in $[19,22]$. It depends on the monitoring of the two considered parameters (i.e., UE speed and RSRP) that exert a high impact on HO performance. The adapted values of HCPs depend on these parameters, which are periodically optimized over time. Algorithm 1 describes the proposed velocity-based optimization algorithm. The initial values of TTT and HOM are set to $100 \mathrm{~ms}$ and $2 \mathrm{~dB}$, respectively.

The algorithm starts with the condition selection according to the measurements of $R S R P_{S}$ and $R S R P_{T}$ pulse the threshold level $\theta_{\text {th }}$, which is assumed to be $2 \mathrm{~dB}$. Then, the $\mathcal{H} \hat{\mathcal{O}} \mathcal{M}$ and $\mathcal{T} \hat{\mathcal{T}} \mathcal{T}$ are adjusted in accordance with the three UE speed states: low, medium, and high. We considered four mobile speeds scenarios: 40, 80,120, and $160 \mathrm{~km} / \mathrm{h}$, where the medium speed is assumed to be $V_{r}$ $=70$ to $90 \mathrm{~km} / \mathrm{h} . \Delta \mathcal{H O} \mathcal{M}_{t}$ and $\Delta \mathcal{T} \mathcal{T} \mathcal{T}_{t}$ represent the adaptive $H O M$ and $T T T$, respectively, where they are updating for each UE in cell to avoid the RLF in each simulation time. $\alpha$ and $\beta$ are the step levels to adapt the values of TTT and HOM in next simulation time which carry values of $50 \mathrm{~ms}$ and 1 $\mathrm{dB}$, respectively. The abovementioned steps are implemented when the HO condition is encountered. Table 2 shows the increase and decrease in the step levels according to mobile speeds. The flowchart of the proposed algorithm is illustrated in Figure 2. 

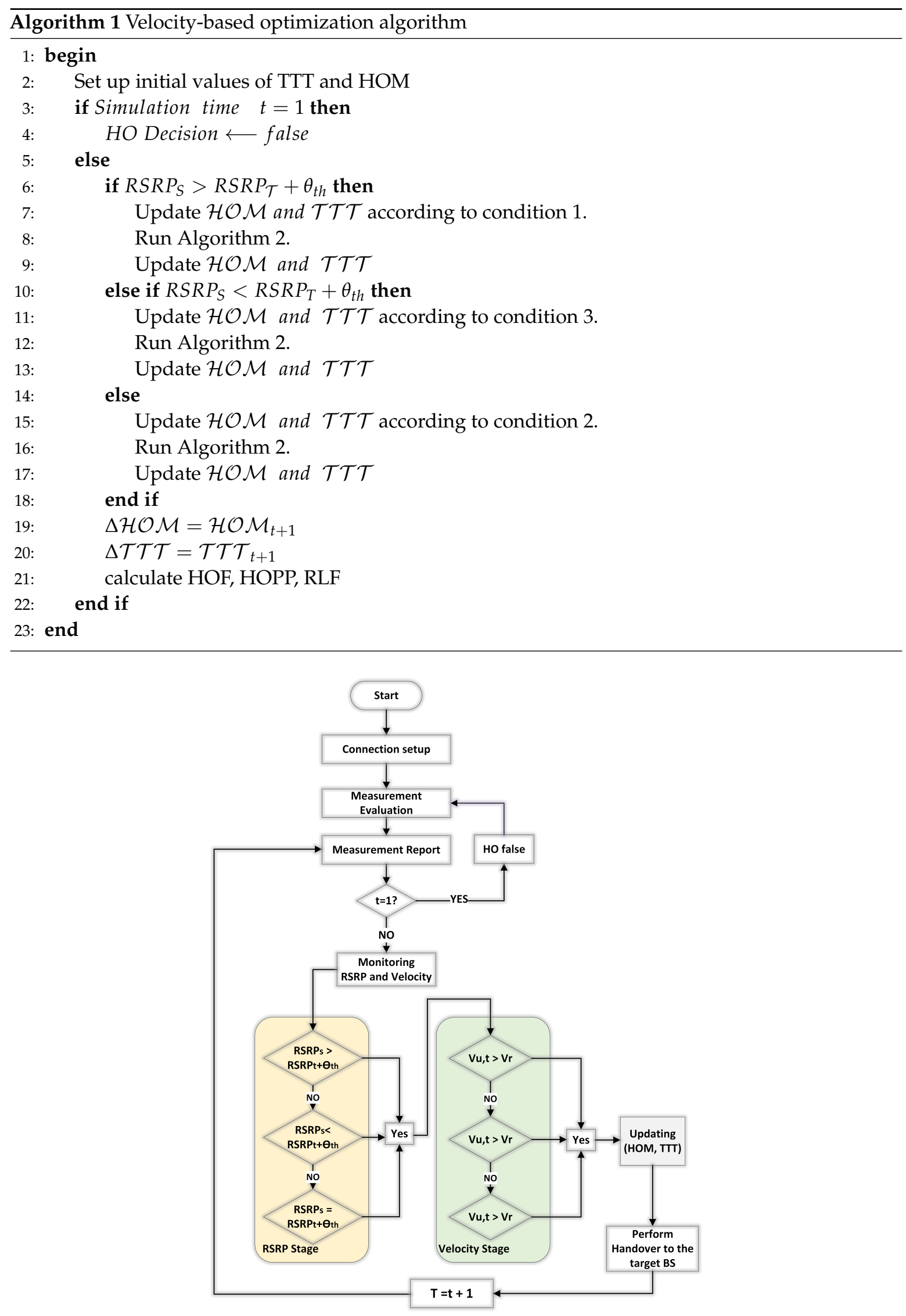

Figure 2. Flowchart of the proposed algorithm. 
Table 2. Adjusting the table of HCPs.

\begin{tabular}{llll}
\hline Conditions & Speeds & $\boldsymbol{\Delta \mathcal { H O } \mathcal { M }}$ & $\boldsymbol{\Delta \mathcal { T } \mathcal { T }}$ \\
\hline \multirow{4}{*}{ Condition 1 } & $V_{u, t}>V_{r}$ & -Step & -Step \\
& $V_{u, t}=V_{r}$ & + Step & +Step \\
& $V_{u, t}<V_{r}$ & +Step & +Step \\
\hline \multirow{4}{*}{ Condition 2 } & $V_{u, t}>V_{r}$ & -Step & -Step \\
& $V_{u, t}=V_{r}$ & None & None \\
& $V_{u, t}<V_{r}$ & +Step & +Step \\
\hline \multirow{3}{*}{ Condition 3 } & $V_{u, t}>V_{r}$ & -Step & -Step \\
& $V_{u, t}=V_{r}$ & -Step & -Step \\
& $V_{u, t}<V_{r}$ & -Step & -Step \\
\hline
\end{tabular}

The UE sends the MR to the serving BS during their mobility in the network. Then, the serving BS checks if the serving RSRP of the UE meets one of the above conditions. If the serving RSRP is greater than the target RSRP plus the threshold $\theta_{t h}$, then the current $\Delta \mathcal{H O} \mathcal{M}_{t}$ and $\Delta \mathcal{T} \mathcal{T} \mathcal{T}_{t}$ are adjusted by adding the current values to the previous values of $\mathcal{H O} \mathcal{M}_{t-1}$ and $\mathcal{T} \mathcal{T} \mathcal{T}_{t-1}$ according to UE speed. After updating the HCPs, the proposed algorithm will check if the HO decision is true or false. Algorithm 2 elaborates the $\mathrm{HO}$ trigger and decision process.

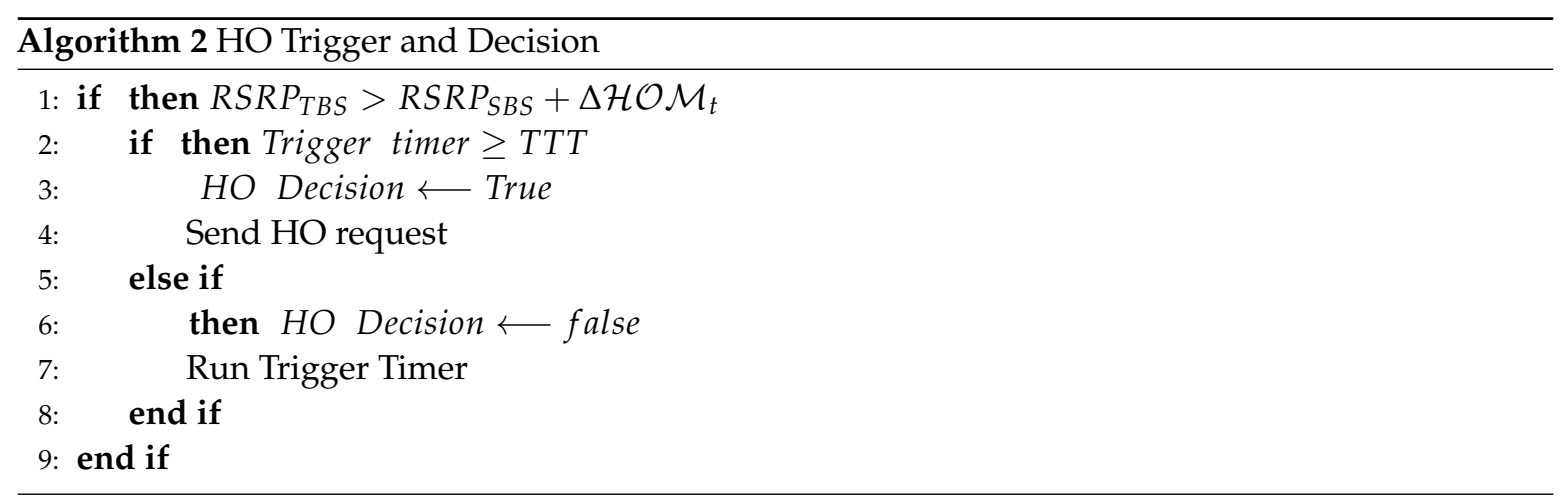

Finally, the main HO performance metrics are calculated and evaluated. The averages of HOP, HOPP, and HOF in each simulation for all UEs are calculated as:

$$
\overline{H O P}=\frac{\sum_{j=1}^{N_{u}} H O P}{N_{u}} \quad \forall j^{t h} U E,
$$

where $N_{u}$ denotes number of UEs overall simulation time. The average of the HOPP per UE are expressed as follows:

$$
\overline{\mathrm{HOPP}}=\frac{N_{\mathrm{HOPP}}}{N_{\mathrm{C}}+N_{F}},
$$

where $N_{H O P P}$ represents the number of HOPPs over the entire simulation. $N_{F}$ and $N_{C}$ represent the number of failed and successful $\mathrm{HO}$, respectively.

RLF is considered when the UEs lose connectivity with the BS during the HO process. The main source of RLF is the failure during HO initialization, which disrupts or causes the radio link to fail. The average probability of the RLF from all UEs is expressed as:

$$
\overline{P(R L F)}=\frac{\sum_{j=1}^{N_{u}} P(R L F)}{N_{u}} \quad \forall j^{\text {th }} U E
$$




\section{Simulation and Performance Analysis}

\subsection{Simulation Scenario}

The performance of the proposed algorithm is evaluated in a realistic cellular communication environment using MATLAB. The simulation environment consists of a two-tier model with multiple small cells that are uniformly distributed within each macro cell coverage [1,22,25]. The network model enables multi-radio access technologies with multi-technology that allow the UEs to connect to one BS. The UEs are randomly distributed in the network and move in random directions within a coverage area. All UEs move with the same speed in four possible directions in each scenario. We consider a line-of-sight connection where no restrictive assumptions are drawn about any obstacles. Table 3 presents the main simulation parameters. As previously mentioned, four mobile speed scenarios are considered to represent the average vehicle speeds in the urban areas.

Table 3. Simulation parameters.

\begin{tabular}{|c|c|c|}
\hline \multirow{2}{*}{ Parameter } & \multicolumn{2}{|r|}{ Value } \\
\hline & 4G Macro Cell & 5G Small Cell \\
\hline Carrier frequency $(\mathrm{GHz})$ & 2.1 & $28[26]$ \\
\hline Number of BS & 61 & 183 \\
\hline Number of UEs/BS & 100 & $200[7]$ \\
\hline Cell radius $(\mathrm{m})$ & 500 & 200 \\
\hline Cell height (m) & 25 & 15 \\
\hline System bandwidth (MHz) & 20 & 500 \\
\hline Transmit power $(\mathrm{dBm})$ & 46 & $30[7]$ \\
\hline Shadowing standard deviation (dB) & 8 & 10 \\
\hline Simulation area $\left(\mathrm{Km}^{2}\right)$ & \multicolumn{2}{|r|}{$8 \times 8$} \\
\hline UE height $(\mathrm{m})$ & \multicolumn{2}{|r|}{1.5} \\
\hline Mobility model & \multicolumn{2}{|r|}{ Random direction model } \\
\hline Noise figure $(\mathrm{dB})$ & \multicolumn{2}{|r|}{9} \\
\hline Thermal noise density $(\mathrm{dBm} / \mathrm{Hz})$ & \multicolumn{2}{|r|}{-174} \\
\hline TTT (ms) & \multicolumn{2}{|r|}{ Adaptive } \\
\hline $\mathrm{HOM}(\mathrm{dB})$ & \multicolumn{2}{|r|}{ Adaptive } \\
\hline HO execution time (ms) & \multicolumn{2}{|r|}{50} \\
\hline
\end{tabular}

\subsection{Performance Evaluation}

\subsubsection{Fixed Values of HCPs}

The HO performance of various fixed HCPs is investigated and validated in this section. The performance represents the average values computed over all UEs in the cells throughout the simulation cycles under various UE speeds. The impact of the different fixed HOM levels on the system performance under different UE speeds are also investigated. The mobility robustness can be investigated using differentiated HOM and TTT settings for all UEs' mobile scenarios as shown Table 4. The performance of the different settings of HCPs is analyzed using two key performance indicators, namely, average HOP and average probability of HOPP.

The effect of user mobility on the system performance is investigated. The average experienced HOP is obtained under different settings of HOM and TTT with respect to different mobile speed scenarios. 
Table 4. Fixed HOM and TTT.

\begin{tabular}{ccc}
\hline \multirow{2}{*}{ Set } & \multicolumn{2}{c}{ HCPs } \\
\cline { 2 - 3 } & HOM (dB) & TTT (ms) \\
\hline Set1 & 5 & 480 \\
Set2 & 10 & 2560 \\
Set3 & 8 & 1500 \\
Set4 & 2 & 512 \\
Set5 & 10 & 320 \\
Set6 & 0 & 40 \\
\hline
\end{tabular}

Figure 3 shows the average HOP over all mobile speeds and simulation times. In general, the reduction in the HOP leads to a significant reduction in the HOPP and the HOF. This phenomenon occurs because at low HOM levels and TTT intervals, UEs can perform early HO to the target cell. By contrast, high HOM level reduces the possibility of UE HO. The result also shows that the Set2 using a relatively high HOM and long TTT $(\mathrm{HOM}=10$, TTT $=2560)$ provides a significant reduction of the average HO probability compared to other settings' overall simulation time. However, the high HOP recorded in Set6 using a low value of HOM and short time of TTT $(\mathrm{HOM}=0$, TTT $=40)$.

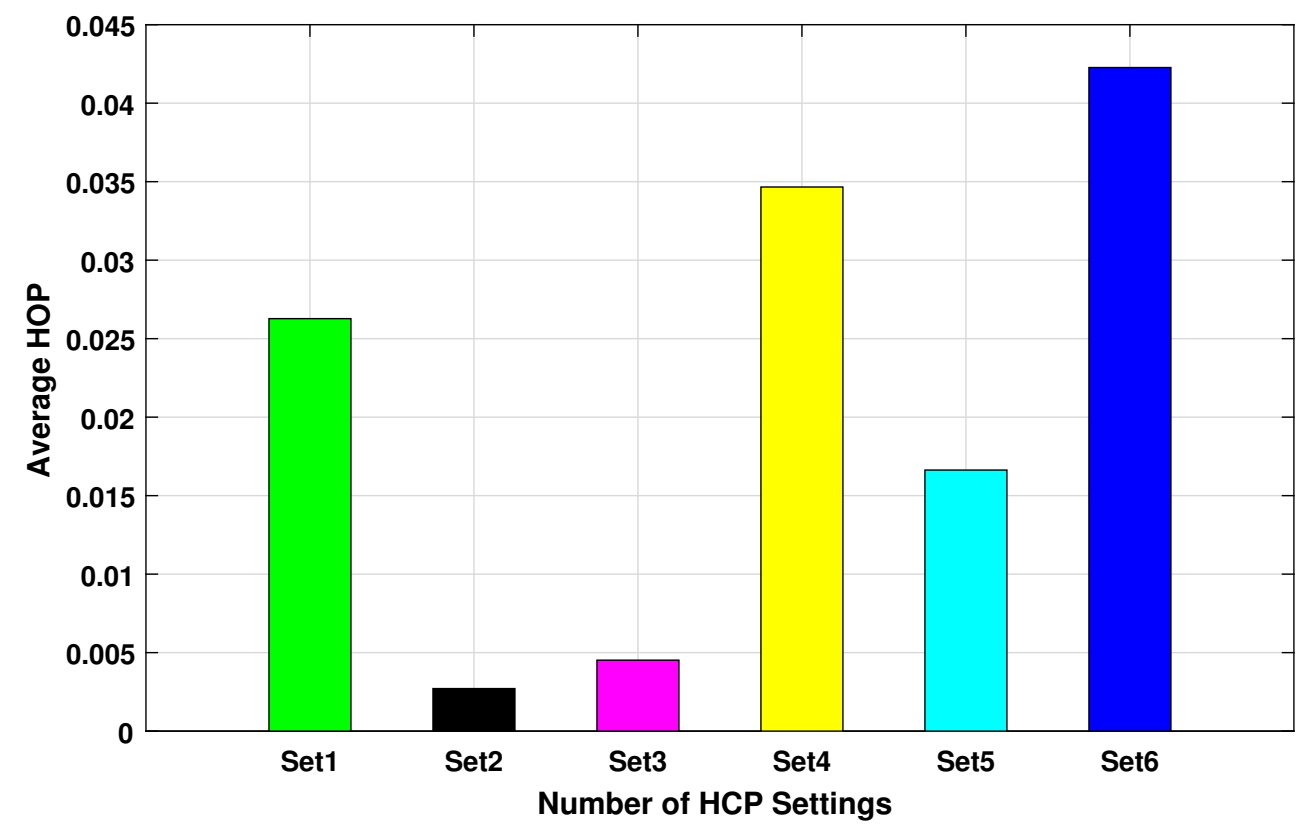

Figure 3. Overall average HOP vs. HCP setting scenarios.

Figures 4 and 5 display the average probability of HOPP versus different mobile speed scenarios. The result shows that the highest achieved rate of HOPP is obtained by Set 6, which can be attributed to the low values of HCPs that cause the UE to bounce between the serving and target BSs. The lowest rate of the HOPP is obtained by Set 2 due to the high values of HCPs.

The behavior of HOP and HOPP with different values of HOMs and TTTs is consistent with the effect of HOM on both parameters. At high TTT intervals, the HOP and HOPP rates decrease and reduce the signaling overhead, whereas at low intervals, both rates increase and raise the signaling overhead. The high UE speed may also cause a high RLF rate because neither the serving BS nor the target BS serves the UE. 

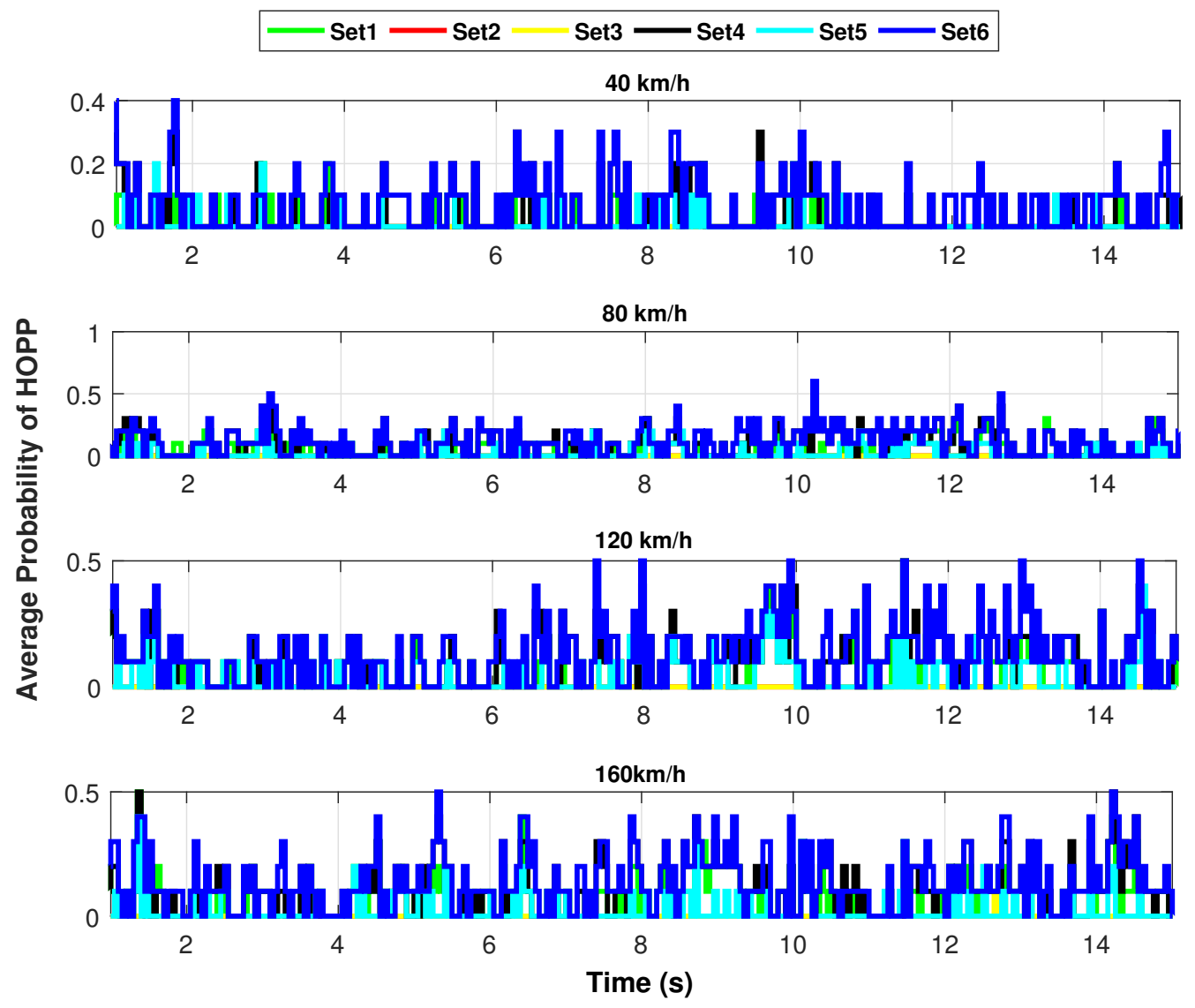

Figure 4. Average probability of HOPP vs. time.

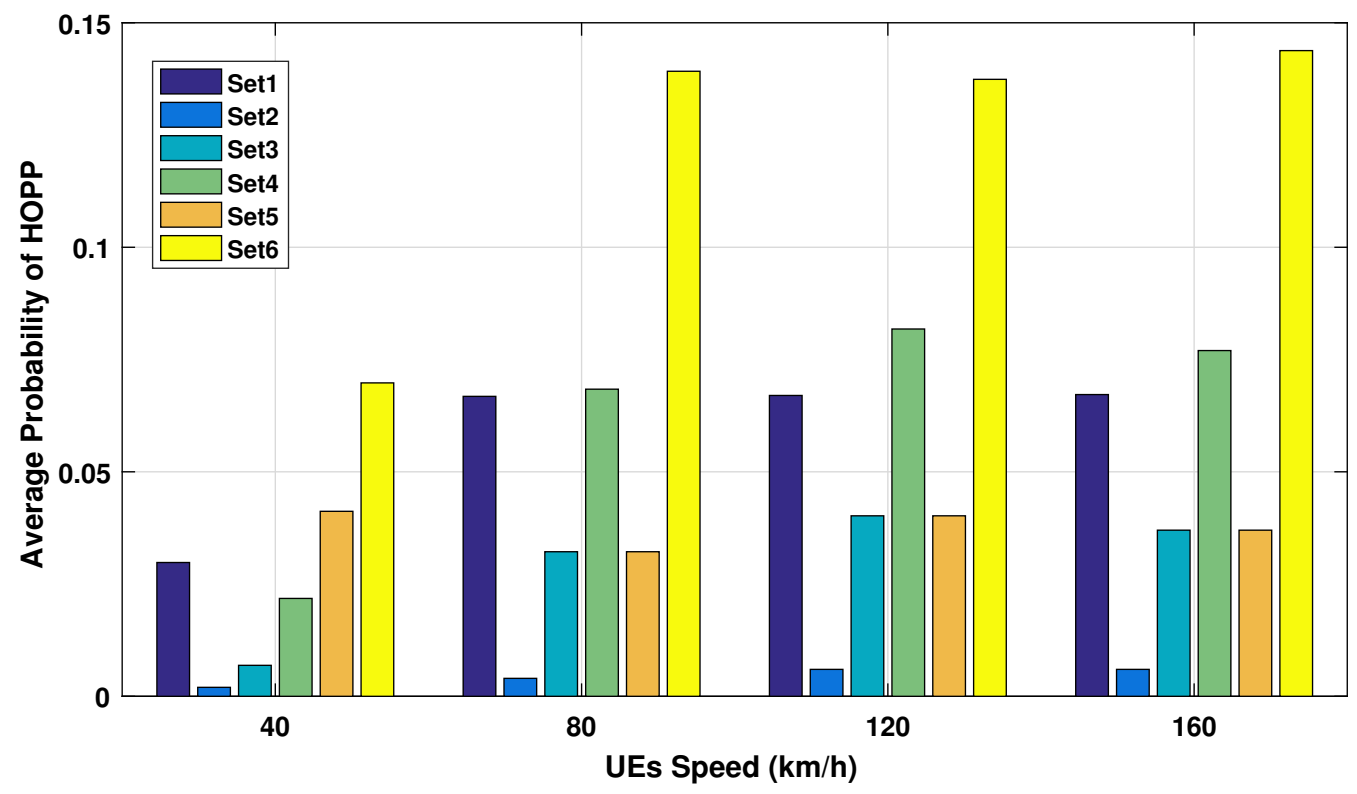

Figure 5. Average probability of HOPP vs. UE speeds.

\subsubsection{Proposed Algorithm}

To analyze the performance of the proposed algorithm, simulations are performed under different mobile speeds. The proposed algorithm is compared with a dynamic algorithm and with those proposed by Ray et al. [11] and Nie et al. [9] algorithms. Figure 6 depicts the average HOP of all 
algorithms under different UE speeds. The proposed algorithm significantly reduces the average HOP compared with the three algorithms under all speeds. The overall average HOP obtained by the proposed algorithm is $70 \%$ less than that obtained by the other algorithms.

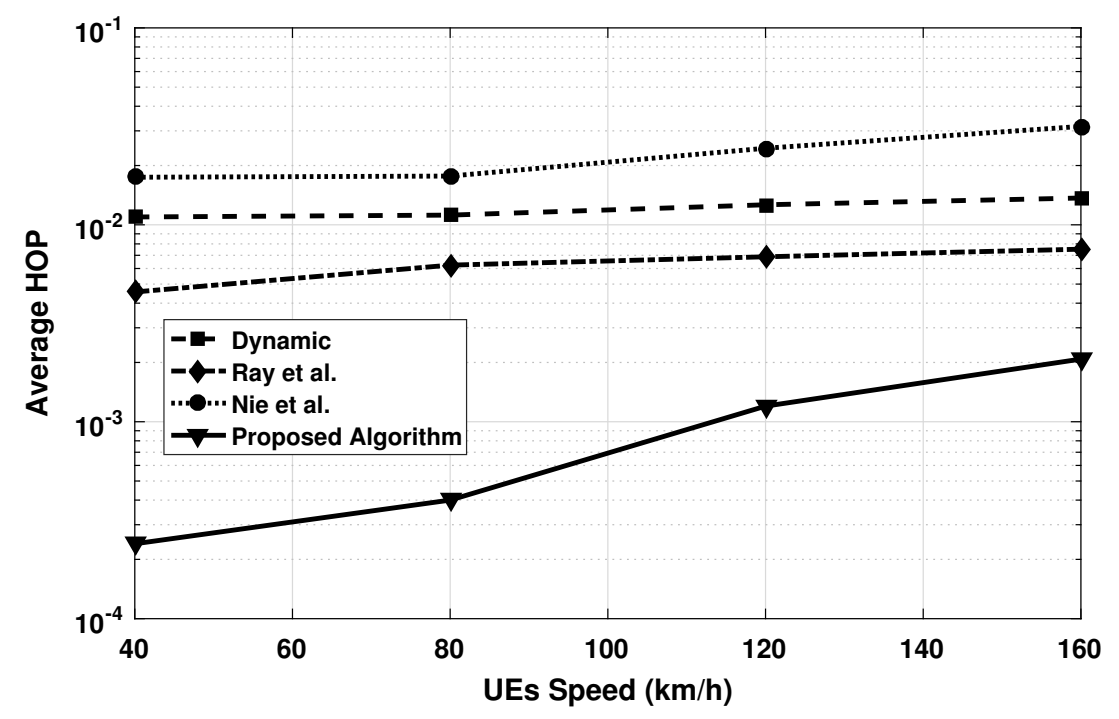

Figure 6. Average HOP with varying UE speeds.

Figure 7 illustrates the average HOPP probability with respect to the UE speeds over the entire simulation time. The simulation results demonstrate that the proposed algorithm obtains a lower HOPP reduction rate than other algorithms under all UE speeds because the other algorithms do not effectively optimize the HCPs according to UE experience, especially when the UEs move near the cell edge. Consequently, the number of UHOs increase due to the high HOPP rate, especially when the UE moves in high speed. Moreover, a high rate of HOP may result in increased HOPP and HOF rates, where a low rate may decrease the HOPP rate.

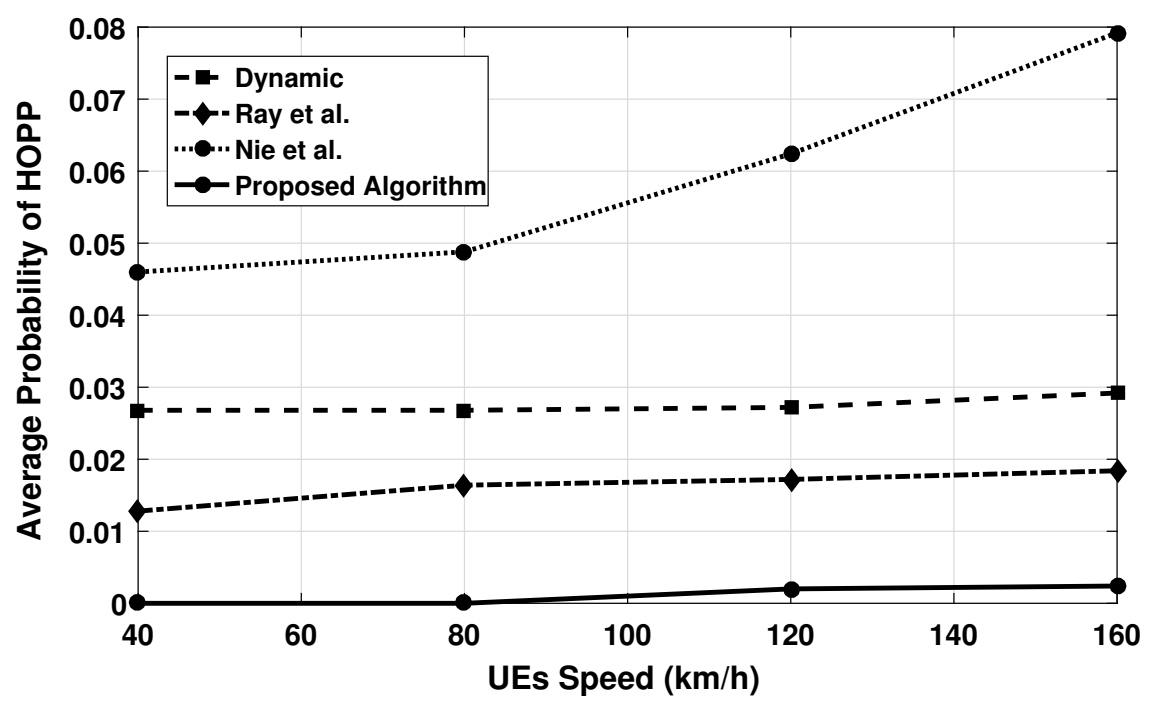

Figure 7. Average probability of HOPP with varying UE speeds.

Figure 8 shows the average probability of RLF with respect to UE mobile speed, which presents the frequent rate of disconnection radio links between BSs and UE mobility. The average probability rate of RLF is obtained from the overall simulation time for each UE speed. The results imply that the 
proposed algorithm achieves a significant reduction in RLF compared with other algorithms under all mobile speed scenarios. The algorithm proposed by Nie et al. achieves a higher RLF rate than other algorithms because only the HOM is adjusted according to UE speed. Therefore, both HCPs should be considered to manage the HO decision according to UE experience. In summary, the RLF rate of all compared algorithms gradually increases when the UE speed increases due to Doppler Effect and weak radio link connections. The proposed algorithm outperforms the other algorithms by more than $70 \%$.

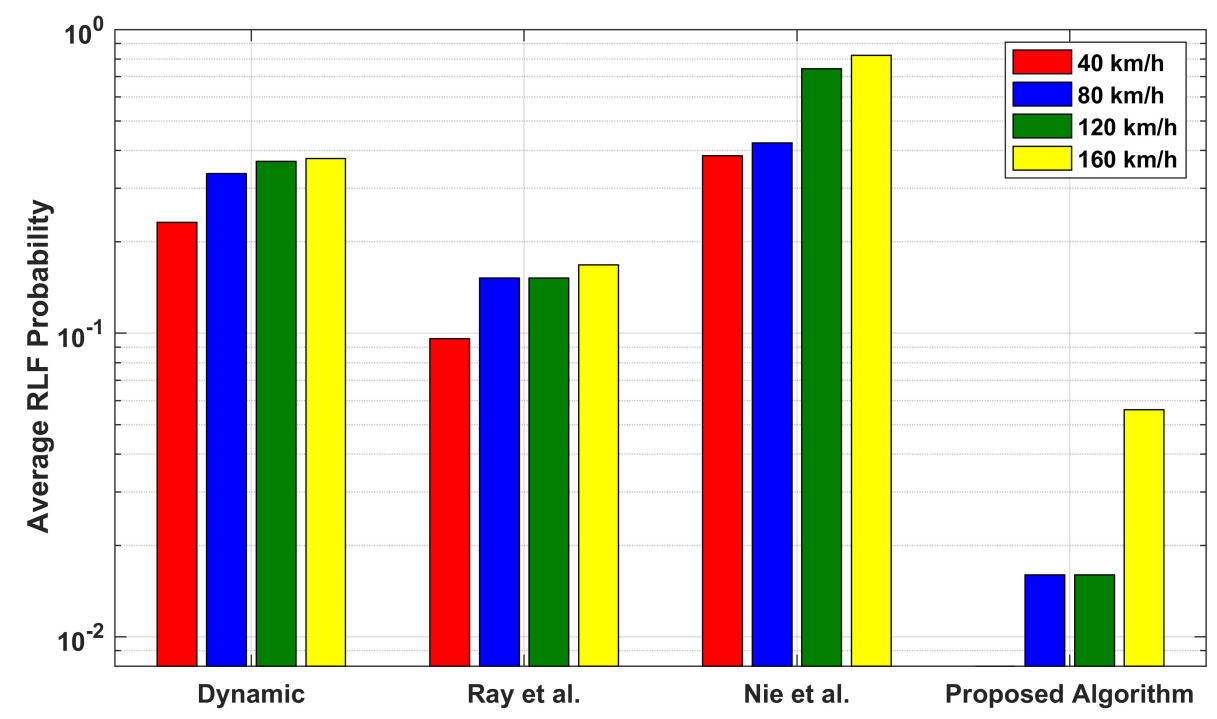

Figure 8. Average probability of RLF for different algorithms.

\section{Conclusions}

In this paper, a velocity-based self-optimization algorithm is proposed to adjust the HCP values in accordance with the UE speed and RSRP. The performances of HO-fixed HCP values and the proposed algorithm are investigated and evaluated. The advantages and disadvantages of the fixed HCPs are highlighted and discussed in terms of the total probability rate of HOP, HOPP, and RLF. The performance of the fixed HCPs results in the introduction of adaptive algorithms, which provides a satisfactory estimation of the HCP values. The performance of the proposed algorithm is analyzed under different mobile speeds and compared with other existing algorithms. The simulation results demonstrate that the proposed algorithm improves the overall system performance under all mobile speed scenarios and outperforms the existing algorithms by more than 70\%. In future research, the proposed algorithm will be further improved by considering the traffic load of the serving BS and the target BS to increase the accuracy of the $\mathrm{HO}$ decision.

Author Contributions: Conceptualization, A.A. (Abdulraqeb Alhammadi), M.R and I.S.; methodology, A.A. (Abdulraqeb Alhammadi), M.R and M.Y.A; software, A.A. (Abdulraqeb Alhammadi), I.S.; validation, A.A. (Abdulraqeb Alhammadi), M.Y.A., and I.S.; formal analysis, A.A. (Abdulraqeb Alhammadi) and I.S.; investigation, A.A. (Abdulraqeb Alhammadi); writing-original draft preparation, A.A. (Abdulraqeb Alhammadi), M.R., I.S., and M.Y.A; writing-review and editing, I.S. and A.A (Abdullah Alquhali); supervision, M.R. and M.Y.A.; project administration, M.R.; funding acquisition, M.R. All authors have read and agreed to the published version of the manuscript.

Funding: This research was funded by Fundamental Research Grant Scheme (FRGS), Grant No. MMUE/170017, Under Ministry of Education, Malaysia.

Conflicts of Interest: The authors declare no conflict of interest. 


\section{Abbreviations}

The following abbreviations are used in this manuscript:

3GPP 3rd generation partnership project

$4 \mathrm{G} \quad$ Fourth generation

5G Fifth generation

AWGN Additive white Gaussian noise

BS Base station

HCP Handover control parameter

HetNets Heterogeneous networks

$\mathrm{HO} \quad$ Handover

HOF Handover failure

HOM Handover margin

HOP Handover probability

HOPP Handover ping-pong

Mmwave Millimeter wave

MR Measurement report

MRO Mobility robustness optimization

NSA Non-standalone

QoS Quality of service

RLF Radio link failure

RSRP Reference signal received power

SA Standalone

SINR Signal-to-noise-ratio

TTT Time to trigger

UDSC Ultra-dense small cell

UE User equipment

UHO Unnecessary handover

\section{References}

1. Universal Mobile Telecommunications System (UMTS), Mobility Enhancementsin Heterogeneous Networks; 3GPP TR 36.839, Tech. Report; 3GPP: Valbonne, France, 2012.

2. Ding, M.; Lopez-Perez, D.; Claussen, H.; Kaafar, M.A. On the fundamental characteristics of ultra-dense small cell networks. IEEE Netw. 2018, 32, 92-100. [CrossRef]

3. Technical Specification Group Services and System Aspects; Release 15 Description; 3GPP TR 21.915, Tech. Report; 3GPP: Valbonne, France, 2019.

4. User Equipment (UE) Radio Transmission and Reception; Part 1: Range 1 Standalone; 3GPP TR 38.101, Tech. Report; 3GPP: Valbonne, France, 2018.

5. Ericsson, H.E. Modified RRH Arrangement for HST SFN; 3GPP: Valbonne, France, 2019.

6. Castro-Hernandez, D.; Paranjape, R. Optimization of Handover Parameters for LTE/LTE-A In-building Systems. IEEE Trans. Veh. Technol. 2017, 67, 5260-5273. [CrossRef]

7. Zhang, H.; Huang, W.; Liu, Y. Handover Probability Analysis of Anchor-Based Multi-Connectivity in 5G User-Centric Network. IEEE Wirel. Commun. Lett. 2018, 8, 396-399. [CrossRef]

8. Evolved Universal Terrestrial Radio Access (e-utra); User Equipment (ue) Procedures in Idle Mode; release 14 (Tech. Rep. No. TS36.304); 3GPP: Valbonne, France, 2018.

9. Nie, S.; Wu, D.; Zhao, M.; Gu, X.; Zhang, L.; Lu, L. An Enhanced Mobility State Estimation Based Handover Optimization Algorithm in LTE-A Self-organizing Network. Procedia Comput. Sci. 2016, 52, 270-277. [CrossRef]

10. Tiwari, R.; Deshmukh, S. Analysis and Design of an Efficient Handoff Management Strategy via Velocity Estimation in HetNets. Trans. Emerg. Telecommun. Technol. 2019, e3642. [CrossRef]

11. Ray, R.P.; Tang, L. Hysteresis Margin and Load Balancing for Handover in Heterogeneous Network. Int. J. Future Comput. Commun. 2016, 4, 231. [CrossRef] 
12. Shayea, I.; Ismail, M.; Nordin, R.; Ergen, M.; Ahmad, N.; Abdullah, N.F.; Alhammadi, A.; Mohamad, H. New weight function for adapting handover margin level over contiguous carrier aggregation deployment scenarios in LTE-advanced system. Wirel. Pers. Commun. 2019, 108, 1179-1199. [CrossRef]

13. Shayea, I.; Ismail, M.; Nordin, R.; Mohamad, H.; Abd Rahman, T.; Abdullah, N.F. Novel Handover Optimization with a Coordinated Contiguous Carrier Aggregation Deployment Scenario in LTE-Advanced Systems. Mob. Infor. Syst. 2016, 2016, 4939872. [CrossRef]

14. Shayea, I.; Ismail, M.; Nordin, R.; Mohamad, H. Adaptive handover decision algorithm based on multi- influence factors through carrier aggregation implementation in LTE-advanced system. J. Comput. Netw. Commun. 2014, 2014, 739504. [CrossRef]

15. Su, D.; Wen, X.; Zhang, H.; Zheng, W. A Self-optimizing Mobility Management Scheme Based on Cell ID Information in High Velocity Environment. In Proceedings of the IEEE Second International Conference on Computer and Network Technology, Bangkok, Thailand, 23-25 April 2010; pp. 285-288.

16. Saeed, M.; Kamal, H.; El-Ghoneimy, M. Novel Type-2 Fuzzy Logic Technique for Handover Problems in a Heterogeneous Network. Eng. Optim. 2018, 50, 1533-1543. [CrossRef]

17. Chaudhuri, S.; Baig, I.; Das, D. Self organizing method for handover performance optimization in LTE-advanced network. Comput. Commun. 2017, 110, 151-163. [CrossRef]

18. Alhammadi, A.; Roslee, M.; Alias, M.Y.; Shayea, I.; Alriah, S.; Abas, A.B. Advanced Handover Self-optimization Approach for 4G/5G HetNets Using Weighted Fuzzy Logic Control. In Proceedings of the 2019 15th International Conference on Telecommunications (ConTEL), Graz, Austria, 3-5 July 2019; pp. 1-6.

19. Alhammadi, A.; Roslee, M.; Alias, M.Y.; Shayea, I.; Alraih, S. Dynamic Handover Control Parameters for LTE-A/5G Mobile Communications. In Proceedings of the 2018 Advances in Wireless and Optical Communications (RTUWO), Riga, Latvia, 15-16 November 2018; pp. 39-44.

20. Abdulraqeb, A.; Mardeni, R.; Yusoff, A.M.; Ibraheem, S.; Saddam, A. Self-optimization of Handover Control Parameters for Mobility Management in 4G/5G Heterogeneous Networks. Autom. Control Comput. Sci. 2019, 53, 441-451. [CrossRef]

21. 5G Study on Channel Model for Frequencies from 0.5 to $100 \mathrm{GHz}$; 3GPP TR 38.901, Tech. Report; 3GPP: Valbonne, France, 2018.

22. Alhammadi, A.; Roslee, M.; Alias, M.Y.; Shayea, I.; Alraih, S.; Mohamed, K.S. Auto Tuning Self-Optimization Algorithm for Mobility Management in LTE-A and 5G HetNets. IEEE Access 2019, 8, 294-304. [CrossRef]

23. Lee, Y.L.; Loo, J.; Chuah, T.C.; Wang, L.C. Dynamic network slicing for multitenant heterogeneous cloud radio access networks. IEEE Trans. Wirel. Commun. 2018, 7, 2146-2161. [CrossRef]

24. Universal Mobile Telecommunications System (UMTS), Radio Resource Control (RRC) Protocol Specification; 3GPP TS 36.331, Tech. Report; 3GPP: Valbonne, France, 2018.

25. Hsieh, P.J.; Lin, W.S.; Lin, K.H.; Wei, H.Y. Dual-connectivity prevenient handover scheme in control/user-plane split networks. IEEE Trans. Veh. Technol. 2018, 67, 3545-3560. [CrossRef]

26. Polese, M.; Giordani, M.; Mezzavilla, M.; Rangan S.; Zorzi, M. Improved Handover Through Dual Connectivity in 5G MmWave Mobile Networks. IEEE J. Sel. Areas Commun. 2017, 35, 2069-2084. [CrossRef]

(C) 2020 by the authors. Licensee MDPI, Basel, Switzerland. This article is an open access article distributed under the terms and conditions of the Creative Commons Attribution (CC BY) license (http://creativecommons.org/licenses/by/4.0/). 